International Journal of Pure and Applied Mathematics

Volume 86 No. 1 2013, 173-197

ISSN: 1311-8080 (printed version); ISSN: 1314-3395 (on-line version)

url: http://www.ijpam.eu

doi: http://dx.doi.org/10.12732/ijpam.v86i1.12

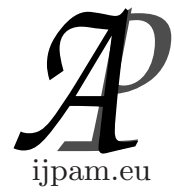

\title{
A DYNAMIC PROBLEM OF FRICTIONLESS CONTACT FOR ELASTIC-THERMO-VISCOPLASTIC MATERIALS WITH DAMAGE
}

\author{
S. Boutechebak \\ Department of Mathematics \\ University of Setif1 \\ 19000, ALGERIA
}

\begin{abstract}
We consider a dynamic frictionless contact problem between an elastic-viscoplastic body and a reactive foundation. The contact is modelled with normal compliance. The material is elastic-viscoplastic with two internal variables which may describe a temperature parameter and the damage of the system caused by plastic deformations. We derive a weak formulation of the system consisting of a motion equation, an energy equation, and an evolution damage inclusion. We prove existence and uniqueness of the solution, and the positivity of the temperature. The proof is based on arguments of nonlinear evolution equations with monotone operators, a classical existence and uniqueness result on parabolic type inequalities, differential equations and fixed-point arguments.
\end{abstract}

AMS Subject Classification: 74H20, 74H25, 74M15, 74F05, 74R20

Key Words: dynamic process, frictionless, contact damage field, subdi ferential, temperature, fixed point

\section{Introduction}

Considerable progress has been achieved recently in modeling, mathematical analysis and numerical simulations of various contact processes. It is concerned

Received: April 29, 2013

(c) 2013 Academic Publications, Ltd. url: www.acadpubl.eu 
with the mathematical structures which underlie general contact problems with different constitutive laws (i.e., different materials), varied geometries and settings, and different contact conditions, see for instance $[3,4,10]$ and the references therein.

The constitutive laws with internal variables has been used in various publications in order to model the effect of internal variables in the behavior of real bodies like metals, rocks polymers and so on, for which the rate of deformation depends on the internal variables. Some of the internal state variables considered by many authors are the spatial display of dislocation, the work-hardening of materials, the absolute temperature and the damage field, see for examples and details $[5,8,25,26,27,28,37,38]$ and references there in for the case of hardening, temperature and other internal state variables and the references $[16,17,18,26,31,34]$ for the case of damage field. Analysis of models for adhesive contact can be found in $[9,10]$, and recently in the monograph [?]. We refer the reader to the extensive bibliography on the subject in $[30,33,35,36]$. The novelty in all the above papers is the introduction of an absolute temperature $\theta$. Then In this paper we extend a part of the results in $[27,35]$ to more general contact conditions for elastic-thermo-viscoplastic materials.

In this paper we deal with the study of a dynamic problem of frictionless contact for general elastic-thermo-viscoplastic materials. For this, we consider a rate-type constitutive equation with two internal variables of the form

$$
\begin{aligned}
\sigma(t)= & \mathcal{A}(\varepsilon(\dot{\mathbf{u}}(t)))+\mathcal{E}(\varepsilon(\mathbf{u}(t))) \\
& +\int_{0}^{t} \mathcal{G}(\sigma(s)-\mathcal{A}(\varepsilon(\dot{\mathbf{u}}(s))), \varepsilon(\mathbf{u}(s)), \theta(s), \varsigma(s)) d s,
\end{aligned}
$$

in which $\mathbf{u}, \sigma$ represent, respectively, the displacement field and the stress field where the dot above denotes the derivative with respect to the time variable, $\theta$ represents the absolute temperature, $\varsigma$ is the damage field, $\mathcal{A}$ and $\mathcal{E}$ are nonlinear operators describing the purely viscous and the elastic properties of the material, respectively, and $\mathcal{G}$ is a nonlinear constitutive function which describes the visco-plastic behavior of the material. Examples and mechanical interpretation of elastic-viscoplastic can be found in $[11,20]$. In this paper, we consider that the material is elastic-viscoplastic with two internal variables which may describe the damage of the system caused by plastic deformations and a temperature parameter. Dynamic and quasistatic contact problems are the topic of numerous papers, e.g. $[1,2,4,13,32]$, and the comprehensive references $[19,36]$. However, the mathematical problem modelled the quasi-static evolution of damage in thermo-viscoplastic materials has been studied in [26]. The paper is organized as follows. In Section 2 we present the mechanical problem 
of the dynamic evolution of damage in elastic-thermo-viscoplastic materials. We introduce some notations and preliminaries and we derive the variational formulation of the problem. We prove in Section 3 the existence and uniqueness of the solution as well as the positivity of the temperature.

\section{Notation and Preliminaries}

In this section we present the notation we shall use and some preliminary material. We denote by $S^{n}$ the space of second order

symmetric tensors on $\mathbb{R}^{n}(n=2,3)$, while "." and | .| will represent the inner product and the Euclidean norm on $S^{n}$ and $\mathbb{R}^{n}$, respectively. Let $\Omega \subset \mathbb{R}^{n}$ $(n=2,3)$ be a bounded domain with a Lipschitz boundary $\Gamma$, partitioned into three disjoint measurable parts $\Gamma_{1}, \Gamma_{2}$ and $\Gamma_{3}$ such that meas $\Gamma_{1}>0$. We denote by $\mathbb{S}_{n}$ the space of symmetric tensors on $\mathbb{R}^{n}$. We define the inner product and the Euclidean norm on $\mathbb{R}^{n}$ and $\mathbb{S}_{n}$, respectively, by

$$
\begin{array}{ccc}
\mathbf{u} \cdot \mathbf{v}=u_{i} v_{i} & \forall \mathbf{u}, \mathbf{v} \in \mathbb{R}^{n}, \quad \sigma \cdot \tau=\sigma_{i j} \tau_{i j} \quad \forall \sigma, \tau \in \mathbb{S}_{n} \\
|\mathbf{u}|=(\mathbf{u} \cdot \mathbf{u})^{1 / 2} & \forall \mathbf{u} \in \mathbb{R}^{n}, \quad|\sigma|=(\sigma \cdot \sigma)^{1 / 2} \quad \forall \sigma \in \mathbb{S}_{n}
\end{array}
$$

Here and below, the indices $i$ and $j$ run from 1 to $n$ and the summation convention over repeated indices is used. We shall use the notation

$$
\begin{gathered}
H=L^{2}(\Omega)^{n}=\left\{\mathbf{u}=\left\{u_{i}\right\}: u_{i} \in L^{2}(\Omega)\right\}, \\
\mathcal{H}=\left\{\sigma=\left\{\sigma_{i j}\right\}: \sigma_{i j}=\sigma_{j i} \in L^{2}(\Omega)\right\}, \\
H_{1}=\{\mathbf{u} \in H: \varepsilon(\mathbf{u}) \in \mathcal{H}\} \\
\mathcal{H}_{1}=\{\sigma \in \mathcal{H}: \operatorname{Div}(\sigma) \in H\}, \\
V=H^{1}(\Omega) .
\end{gathered}
$$

Here $\varepsilon: H_{1} \rightarrow \mathcal{H}$ and Div $: \mathcal{H}_{1} \rightarrow H$ are the deformation and divergence operators, respectively, defined by

$$
\varepsilon(\mathbf{u})=\left(\varepsilon_{i j}(\mathbf{u})\right), \quad \varepsilon_{i j}(\mathbf{u})=\frac{1}{2}\left(u_{i, j}+u_{j, i}\right), \quad \operatorname{Div}(\sigma)=\left(\sigma_{i j, j}\right) .
$$

The sets $H, \mathcal{H}, H_{1}, \mathcal{H}_{1}$ and $V$ are real Hilbert spaces endowed with the canonical inner products:

$$
(\mathbf{u}, \mathbf{v})_{H}=\int_{\Omega} u_{i} v_{i} d x, \quad(\sigma, \tau)_{\mathcal{H}}=\int_{\Omega} \sigma_{i j} \tau_{i j} d x,
$$




$$
\begin{gathered}
(\mathbf{u}, \mathbf{v})_{H_{1}}=(\mathbf{u}, \mathbf{v})_{H}+(\varepsilon(\mathbf{u}), \varepsilon(\mathbf{v}))_{\mathcal{H}}, \\
(\sigma, \tau)_{\mathcal{H}_{1}}=(\sigma, \tau)_{\mathcal{H}}+(\operatorname{Div}(\sigma), \operatorname{Div}(\tau))_{H}, \\
(f, g)_{V}=(f, g)_{L^{2}(\Omega)}+\left(f_{x_{i}}, g_{x_{i}}\right)_{L^{2}(\Omega)} .
\end{gathered}
$$

The associated norms are denoted by $\|\cdot\|_{H},\|\cdot\|_{\mathcal{H}},\|\cdot\|_{H_{1}},\|\cdot\|_{\mathcal{H}_{1}}$ and $\|\cdot\|_{V}$. Let $H_{\Gamma}=\left(H^{1 / 2}(\Gamma)\right)^{n}$ and $\gamma: H_{1} \rightarrow H_{\Gamma}$ be the trace map. We denote by $\mathcal{V}$ the closed subspace of $H_{1}$ defined by

$$
\mathcal{V}=\left\{\mathbf{v} \in H_{1}: \gamma \mathbf{v}=0 \text { on } \Gamma_{1}\right\} .
$$

On the space $\mathcal{V}$ we consider the inner product given by

$$
(\mathbf{u}, \mathbf{v})_{\mathcal{V}}=(\varepsilon(\mathbf{u}), \varepsilon(\mathbf{v}))_{\mathcal{H}}
$$

and let $\|\cdot\|_{\mathcal{V}}$ be the associated norm, defined by

$$
\|\mathbf{v}\|_{\mathcal{V}}=\|\varepsilon(\mathbf{v})\|_{\mathcal{H}}
$$

It follows from Korn's inequality that $\|\cdot\|_{\mathcal{H}_{1}}$ and $\|\cdot\|_{\mathcal{V}}$ are equivalent norms on $V$. Therefore $\left(\mathcal{V},\|\cdot\|_{\mathcal{V}}\right)$ is a real Hilbert space. Moreover, by the Sobolev trace theorem there exists a positive constant $C_{0}$ which depends only on $\Omega, \Gamma_{1}$ and $\Gamma_{3}$ such that

$$
\|\mathbf{v}\|_{L^{2}\left(\Gamma_{3}\right)^{d}} \leq C_{0}\|\mathbf{v}\|_{\mathcal{V}} \quad \forall \mathbf{v} \in \mathcal{V}
$$

The associated norms are denoted by $\|\cdot\|_{H},\|\cdot\|_{\mathcal{H}},\|\cdot\|_{H_{1}},\|\cdot\|_{\mathcal{H}_{1}}$ and $\|\cdot\|_{V}$. Since the boundary $\Gamma$ is Lipschitz continuous, the unit outward normal vector field $\nu$ on the boundary is defined a.e. For every vector field $\mathbf{v} \in H_{1}$ we denote by $v_{\nu}$ and $\mathbf{v}_{\tau}$ the normal and tangential components of $\mathbf{v}$ on the boundary given by

$$
v_{\nu}=\mathbf{v} \cdot \nu, \quad \mathbf{v}_{\tau}=\mathbf{v}-v_{\nu} \nu .
$$

Let $H_{\Gamma}=\left(H^{1 / 2}(\Gamma)\right)^{n}$ and $\gamma: H_{1} \rightarrow H_{\Gamma}$ be the trace map. We denote by $\mathcal{V}$ the closed subspace of $H_{1}$ defined by

$$
\mathcal{V}=\left\{\mathbf{v} \in H_{1}: \gamma \mathbf{v}=0 \text { on } \Gamma_{1}\right\}
$$

We also denote by $H_{\Gamma}^{\prime}$ the dual of $H_{\Gamma}$. Moreover, since meas $\left(\Gamma_{1}\right)>0$, Korn's inequality holds and thus, there exists a positive constant $C_{0}$ depending only on $\Omega, \Gamma_{1}$ such that

$$
\|\varepsilon(\mathbf{v})\|_{\mathcal{H}} \geq C_{0}\|\mathbf{v}\|_{H_{1}} \quad \forall \mathbf{v} \in \mathcal{V} .
$$


On the space $\mathcal{V}$ we consider the inner product given by

$$
(\mathbf{u}, \mathbf{v})_{\mathcal{V}}=(\varepsilon(\mathbf{u}), \varepsilon(\mathbf{v}))_{\mathcal{H}},
$$

and let $\|\cdot\|_{\mathcal{V}}$ be the associated norm, defined by

$$
\|\mathbf{v}\|_{\mathcal{V}}=\|\varepsilon(\mathbf{v})\|_{\mathcal{H}}
$$

It follows from Korn's inequality that $\|\cdot\|_{\mathcal{H}_{1}}$ and $\|\cdot\|_{\mathcal{V}}$ are equivalent norms on $V$. Therefore $(\mathcal{V},\|\cdot\| \mathcal{V})$ is a real Hilbert space. Moreover, by the Sobolev trace theorem there exists a positive constant $C_{0}$ which depends only on $\Omega, \Gamma_{1}$ and $\Gamma_{3}$ such that

$$
\|\mathbf{v}\|_{L^{2}\left(\Gamma_{3}\right)^{d}} \leq C_{0}\|\mathbf{v}\|_{\mathcal{V}} \quad \forall \mathbf{v} \in \mathcal{V}
$$

Furthermore, if $\sigma \in \mathcal{H}_{1}$ there exists an element $\sigma \nu \in H_{\Gamma}^{\prime}$ such that the following Green formula holds

$$
(\sigma, \varepsilon(\mathbf{v}))_{\mathcal{H}}+(\operatorname{Div}(\sigma), \mathbf{v})_{H}=(\sigma \nu, \gamma \mathbf{v})_{H_{\Gamma}^{\prime} \times H_{\Gamma}} \quad \forall \mathbf{v} \in H_{1} .
$$

In addition, if $\sigma$ is sufficiently regular (say $\mathcal{C}^{1}$ ), then

$$
(\sigma, \varepsilon(\mathbf{v}))_{\mathcal{H}}+(\operatorname{Div}(\sigma), \mathbf{v})_{H}=\int_{\Gamma} \sigma \nu \cdot \gamma \mathbf{v} d \gamma \quad \forall \mathbf{v} \in H_{1}
$$

where $d \gamma$ denotes the surface element. Similarly, for a regular tensor field $\sigma: \Omega \rightarrow \mathbb{S}_{n}$ we define its normal and tangential components on the boundary by

$$
\sigma_{\nu}=\sigma \nu \cdot \nu, \quad \sigma_{\tau}=\sigma \nu-\sigma_{\nu} \nu
$$

Moreover, we denote by $\mathcal{V}^{\prime}$ and $V^{\prime}$ the dual of the spaces $\mathcal{V}$ and $V$, respectively. Identifying $H$, respectively $L^{2}(\Omega)$, with its own dual, we have the inclusions

$$
\mathcal{V} \subset H \subset \mathcal{V}^{\prime}, \quad V \subset L^{2}(\Omega) \subset V^{\prime}
$$

We use the notation $\langle\cdot, \cdot\rangle_{\mathcal{V}^{\prime} \times \mathcal{V}},\langle\cdot, \cdot\rangle_{V^{\prime} \times V}$ to represent the duality pairing between $\mathcal{V}^{\prime}, \mathcal{V}$ and $V^{\prime}, V$, respectively.

Let $T>0$. For every real space $X$, we use the notation $C(0, T ; X)$, and $C^{1}(0, T ; X)$ for the space of continuous an continuously differentiable functions from $[0, T]$ to $X$ respectively, $C(0, T ; X)$ is a real Banach space with the norm

$$
|f|_{C(0, T ; X)}=\max _{t \in[0, T]}|f(t)|_{X}
$$

While $C^{1}(0, T ; X)$ is a real Banach space with the norm

$$
|f|_{C^{1}(0, T ; X)}=\max _{t \in[0, T]}|f(t)|_{X}+\max _{t \in[0, T]}|\dot{f}(t)|_{X}
$$


Finally, for $k \in \mathbb{N}$ and $p \in[1, \infty]$, we use the standard notation for the Lebesgue space $L^{p}(0, T ; X)$ and for the Sobolev spaces $W^{k, p}(0, T ; X)$. Moreover for a real number $r$, we use $r_{+}$to represent its positive part that is $r_{+}=\max (0, r)$, and if $X_{1}$ and $X_{2}$ are real Hilbert spaces, than $X_{1} \times X_{2}$ denotes the product Hilbert space endowed with the canonical inner product (., .) $X_{1} \times X_{2}$.

For the rest of this article, we will denote by $C$ possibly different positive constants depending only on the data of the problem, and whose value may change from place to place. Here and in the sequel $\mathrm{C}$ denotes a positive constant which may depend on $\Gamma_{1}, \Gamma_{2}, \Gamma_{3}$, but not depend on $t$ or the initial data.

The physical setting is the following. A body occupies the domain $\Omega$, and is clamped on $\Gamma_{1}$ and so the displacement field vanishes there. Surface tractions of density $\mathbf{f}_{0}$ acts on $\Gamma_{2} \times(0, T)$ and a volume forces of density $\mathbf{f}$ is applied in $\Omega \times(0, T)$. We assume that the body is in adhesive frictionless contact with an obstacle,the so-called foundation, over the potential contact surface $\Gamma_{3}$. we admit a possible external heat source applied in $\Omega \times(0, T)$, given by the function $q$. Moreover, the process is dynamic, and thus the inertial terms are included in the equation of motion. We use an elastic-viscoplastic constitutive law with damage to model the material's behaviour and an ordinary differential equation to describe the evolution of the adhesion field.

The mechanical formulation of the frictionless problem with normal compliance is as follow.

\section{Problem Statement}

We consider an elastic-termo-viscoplastic body which occupies the domain $\Omega \subset$ $\mathbb{R}^{n}$ with the boundary $\Gamma$ divided into three disjoint measurable parts $\Gamma_{1} ; \Gamma_{2}$ and $\Gamma_{3}$ such that meas $\left(\Gamma_{1}\right)>0$. The time interval of interest is $(0 ; T)$ where $T>0$. The body is clamped on $\Gamma_{1}$ and so the displacement field vanishes there. A volume force of density $f$ acts in $\Omega \times(0, T)$ and surface tractions of density $f_{0}$ act on $\Gamma_{2} \times(0, T)$. On the part $\Gamma_{3}$ the body can become in contact with a deformable insulator obstacle and the following normal compliance contact condition is employed:

$$
-\sigma_{\nu}=p\left(u_{\nu}-g\right) \text { on } \Gamma_{3} \times(0, T)
$$

where $\sigma_{\nu}$ is the normal stress, $u_{\nu}$ denotes the normal displacement, $\mathrm{g}$ represents the gap between the body and the obstacle measured along the normal direction $\nu$ and $p$ is a given function whose properties will be described below. Finally, we assume that the contact is frictionless and therefore $\sigma_{\tau}=0$ on $\Gamma_{3} \times(0, T)$. 
Moreover, the process is dynamic, and thus the inertial terms are included in the equation of motion. We use an elastic-thermo- viscoplastic constitutive law with damage to model the material's behaviour and an ordinary differential equation to describe the evolution of the bonding field. Themechanical problem of the dynamic contact of an elastic-thermo-viscoplastic body with a deformable obstacle is then written as follows.

\section{Problem P}

Find the displacement field $\mathbf{u}: \Omega \times(0, T) \rightarrow \mathbb{R}^{n}$, the stress field $\sigma: \Omega \times(0, T) \rightarrow$ $\mathbb{S}_{n}$, the temperature $\theta: \Omega \times(0, T) \rightarrow \mathbb{R}$ and the damage field $\varsigma: \Omega \times(0, T) \rightarrow \mathbb{R}$ such that

$$
\begin{gathered}
\sigma(t)=\mathcal{A}(\varepsilon(\dot{\mathbf{u}}(t)))+\mathcal{E}(\varepsilon(\mathbf{u}(t)))+\int_{0}^{t} \mathcal{G}(\sigma(s) \\
-\mathcal{A}(\varepsilon(\dot{\mathbf{u}}(s))), \varepsilon(\mathbf{u}(s)), \theta(s), \varsigma(s)) d s \quad \text { in } \Omega \text { a.e. } t \in(0, T), \\
\rho \ddot{\mathbf{u}}=\operatorname{Div}(\sigma)+\mathbf{f} \quad \text { in } \Omega \times(0, T), \\
\dot{\theta}-k_{0} \Delta \theta=\psi(\sigma, \varepsilon(\dot{\mathbf{u}}), \theta, \varsigma)+q \quad \text { in } \Omega \times(0, T), \\
\rho \dot{\varsigma}-k_{1} \Delta \varsigma+\partial_{K} \varphi(\varsigma) \quad \phi(\sigma, \varepsilon(\mathbf{u}), \theta, \varsigma) \quad \text { in } \Omega \times(0, T), \\
\mathbf{u}=0 \quad \text { on } \Gamma_{1} \times(0, T), \\
\sigma \nu=\mathbf{f}_{0} \quad \text { on } \Gamma_{2} \times(0, T), \\
-\sigma_{\nu}=p_{\nu}\left(u_{\nu}-g\right) \quad \text { on } \Gamma_{3} \times(0, T), \\
\sigma_{\tau}=0 \quad \text { on } \Gamma_{3} \times(0, T), \\
k_{0} \frac{\partial \theta}{\partial \nu}+\alpha \theta=0 \quad \text { on } \Gamma \times(0, T), \\
\frac{\partial \varsigma}{\partial \nu}=0 \quad \text { on } \Gamma \times(0, T), \\
\dot{\mathbf{u}}(0)=\mathbf{w}_{0}, \quad \theta(0)=\theta_{0}, \quad \varsigma(0)=\varsigma_{0} \quad \text { in } \Omega .
\end{gathered}
$$

This problem represents the dynamic evolution of damage in elastic-thermoviscoplastic materials. Equation (7) is the elastic-thermo-viscoplastic constitutive law where $\mathcal{A}$ and $\mathcal{E}$ are nonlinear operators describing the purely viscous and the elastic properties of the material, respectively, and $\mathcal{G}$ is a nonlinear constitutive function which describes the viscoplastic behavior of the material. (8) represents the equation of motion in which the dot above denotes the derivative with respect to the time variable and $\rho$ is the density of mass. Equation (9) represents the energy conservation where $\psi$ is a nonlinear constitutive function 
which represents the heat generated by the work of internal forces and $q$ is a given volume heat source. Inclusion (10) describes the evolution of damage field, governed by the source damage function $\phi$, where $\partial_{K} \varphi(\varsigma)$ is the subdifferential of indicator function of the set $K$ of admissible damage functions given by

$$
K=\{\xi \in V: 0 \leq \xi(x) \leq 1 \text { a.e. } x \in \Omega\},
$$

in such a way that the damage function $\varsigma$ varied between 0 and 1 . If $\varsigma=1$ there is no damage in the material, if $\varsigma=0$ the material is completely damaged and if $0<\varsigma<1$ the material is partially damaged.

Equalities (11) and (12) are the displacement-traction boundary conditions, respectively. Condition (13) represents the normal compliance condition, $p_{\nu}$ is a given positive function which will be described below. In this condition the interpenetrability between the body and the foundation is allowed. Next, equations (15) and (16) represent, respectively a Fourier boundary condition for the temperature and an homogeneous Neumann boundary condition for the damage field on $\Gamma$. Finally the functions $\mathbf{u}_{0}, \mathbf{w}_{\mathbf{0}}, \theta_{0}$ and $\varsigma_{0}$ in (17) are the initial data.

In the study of the mechanical problem $(\mathrm{P})$, we consider the following hypotheses

The viscosity operator $\mathcal{A}: \Omega \times \mathbb{S}_{n} \rightarrow \mathbb{S}_{n}$ satisfies the following properties:

$$
\begin{aligned}
& \text { (a)There exists a constant } L_{\mathcal{A}}>0 \text { such that } \\
& \left|\mathcal{A}\left(x, \varepsilon_{1}\right)-\mathcal{A}\left(x, \varepsilon_{2}\right)\right| \leq L_{\mathcal{A}}\left|\varepsilon_{1}-\varepsilon_{2}\right| \text { for all } \varepsilon_{1}, \varepsilon_{2} \in \mathbb{S}_{n} \text {, a.e. } x \in \Omega \text {. } \\
& \text { (b)There exists a constant } m_{\mathcal{A}} \text { such that } \\
& \left(\mathcal{A}\left(x, \varepsilon_{1}\right)-\mathcal{A}\left(x, \varepsilon_{2}\right)\right) \cdot\left(\varepsilon_{1}-\varepsilon_{2}\right) \geq m_{\mathcal{A}}\left|\varepsilon_{1}-\varepsilon_{2}\right|^{2} \\
& \text { for all } \varepsilon_{1}, \varepsilon_{2} \in \mathbb{S}_{n} \text { a.e. } x \in \Omega \text {. } \\
& \text { (c) The mapping } x \mapsto \mathcal{A}(x, \varepsilon) \text { is Lebesgue measurable on } \Omega \\
& \text { for all } \varepsilon \in \mathbb{S}_{n} \text {. } \\
& \text { (d) The mapping } x \mapsto \mathcal{A}(x, 0) \in \mathcal{H} \text {. }
\end{aligned}
$$

The elasticity operator $\mathcal{E}: \Omega \times \mathbb{S}_{n} \rightarrow \mathbb{S}_{n}$ satisfies the following properties:

$$
\left\{\begin{array}{l}
\text { (a) There exists a constant } L_{\mathcal{E}}>0 \text { such that } \\
\left|\mathcal{E}\left(x, \varepsilon_{1}\right)-\mathcal{E}\left(x, \varepsilon_{2}\right)\right| \leq L_{\mathcal{E}}\left|\varepsilon_{1}-\varepsilon_{2}\right| \text { for all } \varepsilon_{1}, \varepsilon_{2} \in \mathbb{S}_{n} \text {, a.e. } x \in \Omega . \\
\text { (b)The mapping } x \mapsto \mathcal{E}(x, \varepsilon) \text { is Lebesgue measurable on } \Omega \\
\text { for all } \varepsilon \in \mathbb{S}_{n}, \\
\text { (c)The mapping } x \mapsto \mathcal{E}(x, 0) \in \mathcal{H} .
\end{array}\right.
$$

The viscoplasticity operator $\mathcal{G}: \Omega \times \mathbb{S}_{n} \times \mathbb{S}_{n} \times \mathbb{R} \times \mathbb{R} \rightarrow \mathbb{S}_{n}$ satisifes the following 
properties:

(a)There exists a constant $L_{\mathcal{G}}>0$ such that $\mid \mathcal{G}\left(x, \sigma_{1}, \varepsilon_{1}, \theta_{1}, \varsigma_{1}\right)-$ $\mathcal{G}\left(x, \sigma_{2}, \varepsilon_{2}, \theta_{2}, \varsigma_{2}\right) \mid \leq L_{\mathcal{G}}\left(\left|\sigma_{1}-\sigma_{2}\right|+\left|\varepsilon_{1}-\varepsilon_{2}\right|+\left|\theta_{1}-\theta_{2}\right|+\left|\varsigma_{1}-\varsigma_{2}\right|\right)$ for all $\sigma_{1}, \sigma_{2} \in \mathbb{S}_{n}$, for all $\varepsilon_{1}, \varepsilon_{2} \in \mathbb{S}_{n}$ for all $\theta_{1}, \theta_{2} \in \mathbb{R}$, for all $\varsigma_{1}, \varsigma_{2} \in \mathbb{R}$ a.e. $x \in \Omega$;

(b)The mapping $x \rightarrow \mathcal{G}(\mathbf{x}, \sigma, \varepsilon, \theta, \varsigma)$ is Lebesgue measurable on $\Omega$ for all $\sigma, \varepsilon \in \mathbb{S}_{n}$, for all $\theta, \varsigma \in \mathbb{R}$,

(b)The mapping $x \rightarrow \mathcal{G}(x, 0,0,0,0) \in \mathcal{H}$.

The nonlinear constitutive function $\psi: \Omega \times \mathbb{S}_{n} \times \mathbb{S}_{n} \times \mathbb{R} \times \mathbb{R} \rightarrow \mathbb{R}$ satisfies the following properties:

(a) There exists a constant $L_{\psi}>0$ such that $\mid \psi\left(x, \sigma_{1}, \varepsilon_{1}, \theta_{1}, \varsigma_{1}\right)-$ $\psi\left(x, \sigma_{2}, \varepsilon_{2}, \theta_{2}, \varsigma_{2}\right) \mid \leq L_{\psi}\left(\left|\sigma_{1}-\sigma_{2}\right|+\left|\varepsilon_{1}-\varepsilon_{2}\right|+\left|\theta_{1}-\theta_{2}\right|+\left|\varsigma_{1}-\varsigma_{2}\right|\right)$ for all $\sigma_{1}, \sigma_{2} \in \mathbb{S}_{n}$, for all $\varepsilon_{1}, \varepsilon_{2} \in \mathbb{S}_{n}$, for all $\theta_{1}, \theta_{2} \in \mathbb{R}$, for all $\varsigma_{1}, \varsigma_{2} \in \mathbb{R}$ a.e. $x \in \Omega$,

(b) The mapping $x \rightarrow \psi(x, \sigma, \varepsilon, \theta, \varsigma)$ is Lebesgue measurable on $\Omega$ for all $\sigma, \varepsilon \in \mathbb{S}_{n}$, for all $\theta, \varsigma \in \mathbb{R}$,

(c) The mapping $x \rightarrow \psi(x, 0,0,0,0) \in L^{2}(\Omega)$.

The damage source function $\phi: \Omega \times \mathbb{S}_{n} \times \mathbb{S}_{n} \times \mathbb{R} \times \mathbb{R} \rightarrow \mathbb{R}$ satisfies the following properties:

(a) There exists a constant $L_{\phi}>0$ such that $\left|\phi\left(x, \sigma_{1}, \varepsilon_{1}, \theta_{1}, \varsigma_{1}\right)-\phi\left(x, \sigma_{2}, \varepsilon_{2}, \theta_{2}, \varsigma_{2}\right)\right| \leq L_{\phi}\left(\left|\sigma_{1}-\sigma_{2}\right|+\left|\varepsilon_{1}-\varepsilon_{2}\right|\right.$ $\left.+\left|\theta_{1}-\theta_{2}\right|+\left|\varsigma_{1}-\varsigma_{2}\right|\right)$ for all $\sigma_{1}, \sigma_{2} \in \mathbb{S}_{n}$, for all $\varepsilon_{1}, \varepsilon_{2} \in \mathbb{S}_{n}$, for all $\theta_{1}, \theta_{2} \in \mathbb{R}$, for all $\varsigma_{1}, \varsigma_{2} \in \mathbb{R}$ a.e. $x \in \Omega$

(b) The mapping $x \mapsto \phi(x, \sigma, \varepsilon, \theta, \varsigma)$ is Lebesgue measurable on $\Omega$ for all $\sigma, \varepsilon \in \mathbb{S}_{n}$, for all $\theta, \varsigma \in \mathbb{R}$

(c) The mapping $x \mapsto \phi(x, 0,0,0,0) \in L^{2}(\Omega)$.

The normal compliance function $p: \Gamma_{3} \times \mathbb{R} \longrightarrow \mathbb{R}_{+}$satisfies:

$\left\{\begin{array}{l}\text { (a) There exists a constant } L_{p}>0 \text { such that } \\ \left|p\left(x, r_{1}\right)-p\left(x, r_{2}\right)\right| \leq L_{p}\left|r_{1}-r_{2}\right| \forall r_{1}, r_{2} \in \mathbb{R}, \text { a.e. } x \in \Gamma_{3}, \\ \text { (b) }\left(p\left(x, r_{1}\right)-p\left(x, r_{2}\right)\right)\left(r_{1}-r_{2}\right) \geq 0 \forall r_{1}, r_{2} \in \mathbb{R}, \text { a.e. } x \in \Gamma_{3}, \\ \text { (c) The mapping } x \longrightarrow p(x, r) \text { is measurable on } \Gamma_{3}, \quad \forall r \in \mathbb{R}, \\ \text { (d)The mapping } x \longrightarrow p(x, r)=0 \text { for any } r \leq 0, \text { a.e } x \in \Gamma_{3} .\end{array}\right.$

The mass density satisfies:

$\rho \in L^{\infty}(\Omega)$, there exists $\rho^{*}>0$ such that $\rho \geq \rho^{*}$ a.e. $x \in \Omega$. 
The body forces, surface tractions and the volume heat source have the regularity

$$
\begin{gathered}
\mathbf{f} \in L^{2}(0, T ; H), \quad \mathbf{f}_{0} \in L^{2}\left(0, T ; L^{2}\left(\Gamma_{2}\right)^{n}\right), \\
q \in L^{2}\left(0, T ; L^{2}(\Omega)\right) . \\
\mathbf{u}_{0} \in \mathcal{V}, \quad \mathbf{w}_{0} \in H, \quad \theta_{0} \in V, \quad \varsigma_{0} \in K . \\
k_{i}>0, \quad i=0,1 .
\end{gathered}
$$

We denote by $\mathbf{F}(t) \in \mathcal{V}^{\prime}$ the following element

$$
\langle\mathbf{F}(t), \mathbf{v}\rangle_{\mathcal{V}^{\prime} \times \mathcal{V}}=(\mathbf{f}(t), \mathbf{v})_{H}+\left(\mathbf{f}_{0}(t), \gamma \mathbf{v}\right)_{L^{2}\left(\Gamma_{2}\right)^{n}} \quad \forall \mathbf{v} \in \mathcal{V}, \quad t \in(0, T)
$$

The use of (25) permits to verify that

$$
\mathbf{F} \in L^{2}\left(0, T ; \mathcal{V}^{\prime}\right)
$$

We introduce the following continuous functionals

$$
\begin{gathered}
\mathrm{a}_{0}: V \times V \rightarrow \mathbb{R}, \quad \mathrm{a}_{0}(\zeta, \xi)=k_{0} \int_{\Omega} \nabla \zeta \cdot \nabla \xi d x+\alpha \int_{\Gamma} \zeta \xi d \gamma \\
\mathrm{a}_{1}: V \times V \rightarrow \mathbb{R}, \quad \mathrm{a}_{1}(\zeta, \xi)=k_{1} \int_{\Omega} \nabla \zeta \cdot \nabla \xi d x
\end{gathered}
$$

Finally, we consider the functional $j: \mathcal{V} \times \mathcal{V} \rightarrow \mathbb{R}$ defined by

$$
j(\mathbf{u}, \mathbf{v})=\int_{\Gamma_{3}} p\left(u_{\nu}-g\right) v_{\nu} d a
$$

Keeping in mind (23) and (24), we observe that integrals in (32) are well defined. Using standard arguments based on Green's formula (6), we can derive the following variational formulation of the frictionless problem with normal compliance (7)-(17) as follows.

\section{Problem PV}

Find the displacement field $\mathbf{u}: \Omega \times(0, T) \rightarrow \mathbb{R}^{n}$, the stress field $\sigma: \Omega \times(0, T) \rightarrow$ $\mathbb{S}_{n}$, the temperature $\theta: \Omega \times(0, T) \rightarrow \mathbb{R}$ and the damage field $\varsigma: \Omega \times(0, T) \rightarrow \mathbb{R}$ such that

$$
\begin{aligned}
& \sigma(t)=\mathcal{A}(\varepsilon(\dot{\mathbf{u}}(t)))+\mathcal{E}(\varepsilon(\mathbf{u}(t))) \\
& \quad+\int_{0}^{t} \mathcal{G}(\sigma(s)-\mathcal{A}(\varepsilon(\dot{\mathbf{u}}(s))), \varepsilon(\mathbf{u}(s)), \theta(s), \varsigma(s)) d s \quad \text { a.e. } t \in(0, T),
\end{aligned}
$$




$$
\begin{aligned}
& \langle\rho \ddot{\mathbf{u}}(t), \mathbf{v}\rangle_{\mathcal{V}^{\prime} \times \mathcal{V}}+(\sigma(t), \varepsilon(\mathbf{v}))_{\mathcal{H}}+j(\mathbf{u}(t), \mathbf{v})=\langle\mathbf{F}(t), \mathbf{v}\rangle_{\mathcal{V}^{\prime} \times \mathcal{V}} \\
& \forall \mathbf{v} \in \mathcal{V} \text {, a.e. } t \in(0, T) \text {, } \\
& \langle\rho \dot{\theta}(t), \omega\rangle_{V^{\prime} \times V}+\mathrm{a}_{0}(\theta(t), \omega)=\langle\psi(\sigma(t), \varepsilon(\dot{\mathbf{u}}(t)), \theta(t), \varsigma(t)), \omega\rangle_{V^{\prime} \times V} \\
& +(q(t), \omega)_{L^{2}(\Omega)} \quad \forall \omega \in V \text {, a.e. } t \in(0, T) \text {, } \\
& \langle\rho \dot{\zeta}(t), \xi-\varsigma(t)\rangle_{V^{\prime} \times V}+\mathrm{a}_{1}(\varsigma(t), \xi-\varsigma(t)) \geq\langle\phi(\sigma(t), \varepsilon(\mathbf{u}(t)), \theta(t), \varsigma(t)), \\
& \xi-\varsigma(t)\rangle_{V^{\prime} \times V} \quad \forall \xi \in K \text {, a.e. } t \in(0, T), \varsigma(t) \in K \text {, } \\
& \mathbf{u}(0)=\mathbf{u}_{0}, \quad \dot{\mathbf{u}}(0)=\mathbf{w}_{0}, \quad \theta(0)=\theta_{0}, \quad \varsigma(0)=\varsigma_{0}
\end{aligned}
$$

\section{Main Results}

The existence of the unique solution to Problem PV is proved in the next section. To this end, we consider the following remark which is used in different places of the paper.

Theorem 1 (Existence and uniqueness). Under assumptions (18)-(27), there exists a unique solution $\{\mathbf{u}, \sigma, \theta, \varsigma\}$ to problem $(P V)$. Moreover, the solution has the regularity

$$
\begin{gathered}
\mathbf{u} \in \mathcal{C}^{0}(0, T ; \mathcal{V}) \cap \mathcal{C}^{1}(0, T ; H), \\
\dot{\mathbf{u}} \in L^{2}(0, T ; \mathcal{V}), \\
\ddot{\mathbf{u}} \in L^{2}\left(0, T ; \mathcal{V}^{\prime}\right), \\
\sigma \in L^{2}(0, T ; \mathcal{H}), \\
\theta \in L^{2}(0, T ; V) \cap \mathcal{C}^{0}\left(0, T ; L^{2}(\Omega)\right), \\
\dot{\theta} \in L^{2}\left(0, T ; V^{\prime}\right), \\
\varsigma \in L^{2}(0, T ; V) \cap \mathcal{C}^{0}\left(0, T ; L^{2}(\Omega)\right), \\
\dot{\varsigma} \in L^{2}\left(0, T ; V^{\prime}\right) .
\end{gathered}
$$

A quadruplet $(\mathbf{u}, \sigma, \theta, \varsigma)$ which satisfies (38)-(45) is called a weak solution to the compliance contact problem $\mathbf{P}$. We conclude that under the stated assumptions, problem (7)-(17) has a unique weak solution satisfying (38)-(45). We turn now to the proof of theorem 1 which will be carried out in several steps and is based on arguments of nonlinear equations with monotone operators, a classical existence and uniqueness result on parabolic inequalities and fixed-point arguments. To this end, we assume in the following that (18)(27) hold. Below, $C$ denotes a generic positive constant which may depend 
on $\Omega, \Gamma_{1}, \Gamma_{2}, \Gamma_{3}, \mathcal{A}, \mathcal{E}, \mathcal{G}, \phi, p$ and $T$ but does not depend on $t$ nor of the rest of input data, and whose value may change from place to place. Moreover,for the sake of simplicity we suppress in what follows the explicit dependence of various functions on $x \in \Omega \cup \Gamma$.

Let $\eta \in L^{2}\left(0, T ; \mathcal{V}^{\prime}\right)$ be given. In the first step we consider the following variational problem.

\section{Problem $\mathrm{PV}_{\eta}$}

Find the displacement field $\mathbf{u}_{\eta}: \Omega \times(0, T) \rightarrow \mathbb{R}^{n}$, such that

$$
\begin{gathered}
\left\langle\rho \ddot{\mathbf{u}}_{\eta}(t), \mathbf{v}\right\rangle_{\mathcal{V}^{\prime} \times \mathcal{V}}+\left(\mathcal{A}\left(\varepsilon\left(\dot{\mathbf{u}}_{\eta}(t)\right)\right), \varepsilon(\mathbf{v})\right)_{\mathcal{H}}+\langle\eta(t), \mathbf{v}\rangle_{\mathcal{V}^{\prime} \times \mathcal{V}}=\langle\mathbf{F}(t), \mathbf{v}\rangle_{\mathcal{V}^{\prime} \times \mathcal{V}} \\
\forall \mathbf{v} \in \mathcal{V}, \text { a.e. } t \in(0, T), \\
\mathbf{u}_{\eta}(0)=\mathbf{u}_{0}, \quad \dot{\mathbf{u}}_{\eta}(0)=\mathbf{w}_{0} \text { in } \Omega
\end{gathered}
$$

Lemma 1. For all $\eta \in L^{2}\left(0, T ; \mathcal{V}^{\prime}\right)$, there exists a unique solution $\mathbf{u}_{\eta}$ to the auxiliary problem $P V_{\eta}$ satisfying (38)-(40).

Proof. Let us introduce the operator $A: \mathcal{V} \rightarrow \mathcal{V}^{\prime}$,

$$
\langle A \mathbf{u}, \mathbf{v}\rangle_{\mathcal{V}^{\prime} \times \mathcal{V}}=(\mathcal{A}(\varepsilon(\mathbf{u})), \varepsilon(\mathbf{v}))_{\mathcal{H}}
$$

It follows from (49), (4) and hypothesis (18) that

$$
\|A \mathbf{u}-A \mathbf{v}\|_{\mathcal{V}^{\prime}} \leq L_{\mathcal{A}}\|\mathbf{u}-\mathbf{v}\|_{\mathcal{V}} \quad \forall \mathbf{u}, \mathbf{v} \in \mathcal{V}
$$

Which proves that $A$ is bounded and hemi-continuous on $\mathcal{V}$.

On the other hand, by (4) , (18) and Korn's inequality, we find for every

$$
\frac{\langle A \mathbf{v}, \mathbf{v}\rangle_{\mathcal{V}^{\prime} \times \mathcal{V}}}{\|\mathbf{v}\|_{\mathcal{V}}} \geq C_{0}^{2} m_{\mathcal{A}}\|\mathbf{v}\|_{\mathcal{V}} . \quad \forall \in \mathbf{v} \in \mathcal{V}
$$

The passage to the limit in this inequality when $\|\mathbf{v}\|_{\mathcal{V}} \rightarrow+\infty$ implies that $A$ is coercive in $\mathcal{V}$.

Next, by definition of $A$, the use of (4), (18) and Korn's inequality permits also to obtain

$$
\langle A \mathbf{u}-A \mathbf{v}, \mathbf{u}-\mathbf{v}\rangle_{\mathcal{V}^{\prime} \times \mathcal{V}}>C_{0}^{2} m_{\mathcal{A}}\|\mathbf{u}-\mathbf{v}\|_{\mathcal{V}}^{2} \quad \text { if } \mathbf{u} \neq \mathbf{v}
$$

Then $A$ is strict monotone. Therefore, (47) can be rewritten, making use the operator $A$, as follows

$$
\rho \ddot{\mathbf{u}}_{\eta}(t)+A\left(\dot{\mathbf{u}}_{\eta}(t)\right)=\mathbf{F}_{\eta}(t) \quad \text { on } \mathcal{V}^{\prime} \text { a.e. } t \in(0, T),
$$


where

$$
\mathbf{F}_{\eta}(t)=\mathbf{F}(t)-\eta(t) \in \mathcal{V}^{\prime}
$$

We recall that by $(29)$ we have $\mathbf{F}_{\eta} \in L^{2}\left(0, T ; \mathcal{V}^{\prime}\right)$. Kipping in mind that the operator $A$ is strict monotone, hemi-continuous, bounded and coercive, then by using classical arguments of functional analysis concerning parabolic equations $[7,23]$ we can easily prove the existence and uniqueness of $\mathbf{w}_{\eta}$ satisfying

$$
\begin{gathered}
\mathbf{w}_{\eta} \in L^{2}(0, T ; \mathcal{V}) \cap \mathcal{C}^{0}(0, T ; H) \\
\dot{\mathbf{w}}_{\eta} \in L^{2}\left(0, T ; \mathcal{V}^{\prime}\right) \\
\rho \dot{\mathbf{w}}_{\eta}(t)+A\left(\mathbf{w}_{\eta}(t)\right)=\mathbf{F}_{\eta}(t) \text { on } \mathcal{V}^{\prime} \text { a.e. } t \in(0, T), \\
\mathbf{w}_{\eta}(0)=\mathbf{w}_{0}
\end{gathered}
$$

Consider now the function $\mathbf{u}_{\eta}:(0, T) \rightarrow \mathcal{V}$ defined by

$$
\mathbf{u}_{\eta}(t)=\int_{0}^{t} \mathbf{w}_{\eta}(s) d s+\mathbf{u}_{0} \quad \forall t \in(0, T) .
$$

It follows from (53) and (54) that $\mathbf{u}_{\eta}$ is a solution of the equation (50) and it satisfies (38)-(40).

In the second step we use the displacement field $u_{\eta}$ obtained in Lemma1 and we consider the following initial-value problem.

\section{Problem $\mathrm{PV}_{\boldsymbol{\lambda}}$}

Find the temperature $\theta_{\lambda}: \Omega \times(0, T) \rightarrow \mathbb{R}$ such that

$$
\begin{gathered}
\left\langle\rho \dot{\theta}_{\lambda}(t), \omega\right\rangle_{V^{\prime} \times V}+\mathrm{a}_{0}\left(\theta_{\lambda}(t), \omega\right)=\langle\lambda(t)+q(t), \omega\rangle_{V^{\prime} \times V} \\
\forall \omega \in V \text {, a.e. } t \in(0, T), \\
\theta_{\lambda}(0)=\theta_{0} \text { in } \Omega .
\end{gathered}
$$

Lemma 2. For all $\lambda \in L^{2}\left(0, T ; V^{\prime}\right)$, there exists a unique solution $\theta_{\lambda}$ to the auxiliary problem $P V_{\lambda}$ satisfying (42) and (43).

Proof. By an application of the Poincaré-Friedrichs inequality, we can find a constant $\alpha^{\prime}>0$ such that

$$
\int_{\Omega}|\nabla \zeta|^{2} d x+\frac{\alpha}{k_{0}} \int_{\Gamma}|\zeta|^{2} d \gamma \geq \alpha^{\prime} \int_{\Omega}|\zeta|^{2} d x \quad \forall \zeta \in V .
$$


Thus, we obtain

$$
\mathrm{a}_{0}(\zeta, \zeta) \geq C_{1}\|\zeta\|_{V}^{2} \quad \forall \zeta \in V
$$

where $C_{1}=k_{0} \min \left(1, \alpha^{\prime}\right) / 2$, which implies that $\mathrm{a}_{0}$ is $V$-elliptic. Consequently, based on classical arguments of functional analysis concerning parabolic equations, the variational equation (56) has a unique solution $\theta_{\lambda}$ satisfies (42) and $(43)$.

\section{Problem $\mathrm{PV}_{\mu}$}

Find the damage field $\varsigma_{\mu}: \Omega \times(0, T) \rightarrow \mathbb{R}$ such that

$$
\begin{gathered}
\left\langle\rho \dot{\varsigma}_{\mu}(t), \xi-\varsigma_{\mu}(t)\right\rangle_{V^{\prime} \times V}+\mathrm{a}_{1}\left(\varsigma_{\mu}(t), \xi-\varsigma_{\mu}(t)\right) \\
\geq\left\langle\mu, \xi-\varsigma_{\mu}(t)\right\rangle_{V^{\prime} \times V} \quad \forall \xi \in K, \text { a.e. } t \in(0, T), \varsigma_{\mu}(t) \in K, \\
\varsigma_{\mu}(0)=\varsigma_{0} \quad \text { in } \Omega .
\end{gathered}
$$

Lemma 3. For all $\mu \in L^{2}\left(0, T ; V^{\prime}\right)$, there exists a unique solution $\varsigma_{\mu}$ to the auxiliary problem $P V_{\mu}$ satisfying (44)-(45).

Proof. We know that the form a $a_{1}$ is not $V$-elliptic. To solve this problem we introduce the functions

$$
\tilde{\varsigma}_{\mu}(t)=e^{-k_{1} t} \varsigma_{\mu}(t), \quad \tilde{\xi}(t)=e^{-k_{1} t} \xi(t)
$$

We remark that if $\varsigma_{\mu}, \xi \in K$ then $\tilde{\varsigma}_{\mu}, \tilde{\xi} \in K$. Consequently, (59) is equivalent to the inequality

$$
\begin{aligned}
& \left\langle\rho \tilde{\varsigma}_{\mu}(t), \tilde{\xi}-\tilde{\varsigma}_{\mu}(t)\right\rangle_{V^{\prime} \times V}+\mathrm{a}_{1}\left(\tilde{\varsigma}_{\mu}(t), \tilde{\xi}-\tilde{\varsigma}_{\mu}(t)\right)+k_{1}\left(\rho \tilde{\varsigma}_{\mu}, \tilde{\xi}-\tilde{\varsigma}_{\mu}(t)\right)_{L^{2}(\Omega)} \\
& \geq\left\langle e^{-k_{1} t} \mu, \tilde{\xi}-\tilde{\varsigma}_{\mu}(t)\right\rangle_{V^{\prime} \times V} \quad \forall \tilde{\xi} \in K, \text { a.e. } t \in(0, T), \tilde{\varsigma}_{\mu} \in K .
\end{aligned}
$$

The fact that

$$
\mathrm{a}_{1}(\tilde{\xi}, \tilde{\xi})+k_{1}(\rho \tilde{\xi}, \tilde{\xi})_{L^{2}(\Omega)} \geq k_{1} \min \left(\rho^{*}, 1\right)\|\tilde{\xi}\|_{V}^{2} \quad \forall \tilde{\xi} \in V
$$

and using classical arguments of functional analysis concerning parabolic inequalities $[7,12]$, implies that $(59)$ has a unique solution $\tilde{\varsigma}_{\mu}$ having the regularity (44) and (45).

Let us consider now the auxiliary problem. 


\section{Problem $\mathrm{PV}_{\eta, \lambda, \mu}$}

Find the stress field $\sigma_{\eta, \lambda, \mu}: \Omega \times(0, T) \rightarrow \mathbb{S}_{n}$ which is a solution of the problem

$$
\begin{aligned}
\sigma_{\eta, \lambda, \mu}(t)= & \mathcal{E}\left(\varepsilon\left(\mathbf{u}_{\eta}(t)\right)\right)+\int_{0}^{t} \mathcal{G}\left(\sigma_{\eta, \lambda, \mu}(s)\right. \\
& \left.-\mathcal{A}\left(\varepsilon\left(\dot{\mathbf{u}}_{\eta}(s)\right)\right), \varepsilon\left(\mathbf{u}_{\eta}(s)\right), \theta_{\lambda}(s), \varsigma_{\mu}(s)\right) d s \quad \text { a.e. } t \in(0, T),
\end{aligned}
$$

Lemma 4. There exists a unique solution of Problem $P V_{\eta, \lambda, \mu}$ and it satisfies (41). Moreover, if $\mathbf{u}_{\eta_{i}}, \theta_{\lambda_{i}}, \varsigma_{\mu_{i}}$ and $\sigma_{\eta_{i}, \lambda_{i}, \mu_{i}}$ represent the solutions of problems $P V_{\eta_{i}}, P V_{\lambda_{i}}, P V_{\mu_{i}}$ and $P V_{\eta_{i}, \lambda_{i}, \mu_{i}}$, respectively, for $i=1,2$, then there exists $C>0$ such that

$$
\begin{aligned}
& \left\|\sigma_{\eta_{1}, \lambda_{1}, \mu_{1}}(t)-\sigma_{\eta_{2}, \lambda_{2}, \mu_{2}}(t)\right\|_{\mathcal{H}}^{2} \\
& \leq C \int_{0}^{t}\left(\left\|\dot{\mathbf{u}}_{\eta_{1}}(s)-\dot{\mathbf{u}}_{\eta_{2}}(s)\right\|_{\mathcal{V}}^{2}+\left\|\mathbf{u}_{\eta_{1}}(s)-\mathbf{u}_{\eta_{2}}(s)\right\|_{\mathcal{V}}^{2}\right. \\
& \left.\quad+\left\|\theta_{\lambda_{1}}(s)-\theta_{\lambda_{2}}(s)\right\|_{V}^{2}+\left\|\varsigma_{\mu_{1}}(s)-\varsigma_{\mu_{2}}(s)\right\|_{V}^{2}\right) d s
\end{aligned}
$$

Proof. Let $\Sigma_{\eta, \lambda, \mu}: L^{2}(0, T ; \mathcal{H}) \rightarrow L^{2}(0, T ; \mathcal{H})$ be the mapping given by

$$
\begin{aligned}
& \Sigma_{\eta, \lambda, \mu} \sigma(t) \\
& =\mathcal{E}\left(\varepsilon\left(\mathbf{u}_{\eta}(t)\right)\right)+\int_{0}^{t} \mathcal{G}\left(\sigma(s)-\mathcal{A}\left(\varepsilon\left(\dot{\mathbf{u}}_{\eta}(s)\right)\right), \varepsilon\left(\mathbf{u}_{\eta}(s)\right), \theta_{\lambda}(s), \varsigma_{\mu}(s)\right) d s .
\end{aligned}
$$

Let $\sigma_{i} \in L^{2}(0, T ; \mathcal{H}), i=1,2$ and $t_{1} \in(0, T)$. We find be using hypothesis $(20)$ and Hölder's inequality

$$
\left\|\Sigma_{\eta, \lambda, \mu} \sigma_{1}\left(t_{1}\right)-\Sigma_{\eta, \lambda, \mu} \sigma_{2}\left(t_{1}\right)\right\|_{\mathcal{H}}^{2} \leq L_{\mathcal{G}}^{2} T \int_{0}^{t_{1}}\left\|\sigma_{1}(s)-\sigma_{2}(s)\right\|_{\mathcal{H}}^{2} d s
$$

By reapplication of mapping $\Sigma_{\eta, \lambda, \mu}$, it follows that

$$
\| \Sigma_{\eta, \lambda, \mu}^{2} \sigma_{1}\left(t_{1}-\Sigma_{\eta, \lambda, \mu}^{2} \sigma_{2}\left(t_{1}\right)\left\|_{\mathcal{H}}^{2} \leq L_{\mathcal{G}}^{4} T^{2} \int_{0}^{t_{1}} \int_{0}^{t_{2}}\right\| \sigma_{1}(s)-\sigma_{2}(s) \|_{\mathcal{H}}^{2} d s d t_{2}\right.
$$

Reiterating this inequality $n$ times leads to

$$
\left\|\Sigma_{\eta, \lambda, \mu}^{n} \sigma_{1}\left(t_{1}\right)-\Sigma_{\eta, \lambda, \mu}^{n} \sigma_{2}\left(t_{1}\right)\right\|_{\mathcal{H}}^{2} \leq L_{\mathcal{G}}^{2 n} T^{n} \int_{0}^{t_{1}} \int_{0}^{t_{2}} \ldots \int_{0}^{t_{n}}\left\|\sigma_{1}(s)-\sigma_{2}(s)\right\|_{\mathcal{H}}^{2} d s d t_{n} \ldots d t_{2} .
$$


Integration on the time interval $(0, T)$, it follows that

$$
\left\|\Sigma_{\eta, \lambda, \mu}^{n} \sigma_{1}-\Sigma_{\eta, \lambda, \mu}^{n} \sigma_{2}\right\|_{L^{2}(0, T ; \mathcal{H})}^{2} \leq \frac{L_{\mathcal{G}}^{2 n} T^{2 n}}{n !}\left\|\sigma_{1}-\sigma_{2}\right\|_{L^{2}(0, T ; \mathcal{H})}^{2}
$$

It follows from this inequality that for $n$ large enough, a power $n$ of the mapping $\Sigma_{\eta, \lambda, \mu}$ is a contraction on the space $L^{2}(0, T ; \mathcal{H})$ and, therefore, from the Banach fixed point theorem, there exists a unique element $\sigma_{\eta, \lambda, \mu} \in L^{2}(0, T ; \mathcal{H})$ such that $\Sigma_{\eta, \lambda, \mu} \sigma_{\eta, \lambda, \mu}=\sigma_{\eta, \lambda, \mu}$, which represents the unique solution of the problem $\mathrm{PV}_{\eta, \lambda, \mu}$. Moreover, if $\mathbf{u}_{\eta_{i}}, \theta_{\lambda_{i}}, \varsigma_{\mu_{i}}$ and $\sigma_{\eta_{i}, \lambda_{i}, \mu_{i}}$ represent the solutions of the problems $\mathrm{PV}_{\eta_{i}}, \mathrm{PV}_{\lambda_{i}}, \mathrm{PV}_{\mu_{i}}$ and $\mathrm{PV}_{\eta_{i}, \lambda_{i}, \mu_{i}}$, respectively, for $i=1,2$, then we use (18)-(20) and Young's inequality to obtain

$$
\begin{aligned}
& \left\|\sigma_{\eta_{1}, \lambda_{1}, \mu_{1}}(t)-\sigma_{\eta_{2}, \lambda_{2}, \mu_{2}}(t)\right\|_{\mathcal{H}}^{2} \\
& \leq C\left\|\sigma_{\eta_{1}, \lambda_{1}, \mu_{1}}(t)-\sigma_{\eta_{2}, \lambda_{2}, \mu_{2}}(t)\right\|_{\mathcal{H}}^{2}+C \int_{0}^{t}\left(\left\|\dot{\mathbf{u}}_{\eta_{1}}(s)-\dot{\mathbf{u}}_{\eta_{2}}(s)\right\|_{\mathcal{V}}^{2}\right. \\
& \left.\quad+\left\|\mathbf{u}_{\eta_{1}}(s)-\mathbf{u}_{\eta_{2}}(s)\right\|_{\mathcal{V}}^{2}+\left\|\theta_{\lambda_{1}}(s)-\theta_{\lambda_{2}}(s)\right\|_{V}^{2}+\left\|\varsigma_{\mu_{1}}(s)-\varsigma_{\mu_{2}}(s)\right\|_{V}^{2}\right) d s .
\end{aligned}
$$

Which permits us to obtain, using Gronwall's lemma, the inequality (64). Second step. Let us consider the mapping

$$
\Lambda: L^{2}\left(0, T ; \mathcal{V}^{\prime} \times V^{\prime} \times V^{\prime} \rightarrow L^{2}\left(0, T ; \mathcal{V}^{\prime} \times V^{\prime} \times V^{\prime}\right)\right.
$$

defined by

$$
\begin{aligned}
& \Lambda(\eta(t), \lambda(t), \mu(t)) \\
& =\left(\Lambda_{0}(\eta(t), \lambda(t), \mu(t)), \psi\left(\sigma_{\eta, \lambda, \mu}(t), \varepsilon\left(\dot{\mathbf{u}}_{\eta}(t)\right), \theta_{\lambda}(t), \varsigma_{\mu}(t)\right),\right. \\
& \left.\quad \phi\left(\sigma_{\eta, \lambda, \mu}(t), \varepsilon\left(\mathbf{u}_{\eta}(t)\right), \theta_{\lambda}(t), \varsigma_{\mu}(t)\right)\right),
\end{aligned}
$$

where the mapping $\Lambda_{0}$ is given by

$$
\begin{aligned}
& \left\langle\Lambda_{0}(\eta(t), \lambda(t), \mu(t)), \mathbf{v}\right\rangle_{\mathcal{V}^{\prime} \times \mathcal{V}} \\
& =\left(\mathcal{E}\left(\varepsilon\left(\mathbf{u}_{\eta}(t)\right)\right)+\int_{0}^{t} \mathcal{G}\left(\sigma_{\eta, \lambda, \mu}(s) \quad-\mathcal{A}\left(\varepsilon\left(\dot{\mathbf{u}}_{\eta}(s)\right)\right), \varepsilon\left(\mathbf{u}_{\eta}(s)\right),\right.\right. \\
& \left.\left.\theta_{\lambda}(s), \varsigma_{\mu}(s)\right) d s, \varepsilon(\mathbf{v})\right)_{\mathcal{H}}+j\left(\mathbf{u}_{\eta}(t), \mathbf{v}\right) \quad \forall \mathbf{v} \in \mathcal{V} .
\end{aligned}
$$

Lemma 5. The mapping $\Lambda$ has a fixed point

$$
\left(\eta^{*}, \lambda^{*}, \mu^{*}\right) \in L^{2}\left(0, T ; \mathcal{V}^{\prime} \times V^{\prime} \times V^{\prime}\right) .
$$


Proof. Let $t \in(0, T)$ and

$$
\left(\eta_{1}, \lambda_{1}, \mu_{1}\right),\left(\eta_{2}, \lambda_{2}, \mu_{2}\right) \in L^{2}\left(0, T ; \mathcal{V}^{\prime} \times V^{\prime} \times V^{\prime}\right)
$$

We use the notation $\mathbf{u}_{\eta_{i}}=\mathbf{u}_{i}, \dot{\mathbf{u}}_{\eta_{i}}=\dot{\mathbf{u}}_{i}, \ddot{\mathbf{u}}_{\eta_{i}}=\ddot{\mathbf{u}}_{i}, \theta_{\lambda_{i}}=\theta_{i}, \varsigma_{\mu_{i}}=\varsigma_{i}$ and $\sigma_{\eta_{i}, \lambda_{i}, \mu_{i}}=\sigma_{i}$, for $i=1,2$. Let us start by using (5)and hypotheses (18), (19),(20), (22), to obtain

$$
\begin{aligned}
& \left\|\Lambda_{0}\left(\eta_{1}(t), \lambda_{1}(t), \mu_{1}(t)\right)-\Lambda_{0}\left(\eta_{2}(t), \lambda_{2}(t), \mu_{2}(t)\right)\right\|_{\mathcal{V}^{\prime}} \\
& \leq L_{\mathcal{E}}\left\|\mathbf{u}_{1}(t)-\mathbf{u}_{2}(t)\right\|_{\mathcal{V}}+L_{\mathcal{G}} \int_{0}^{t}\left(\left\|\sigma_{1}(s)-\sigma_{2}(s)\right\|_{\mathcal{H}}+L_{\mathcal{A}}\left\|\dot{\mathbf{u}}_{1}(s)-\dot{\mathbf{u}}_{2}(s)\right\|_{\mathcal{V}}\right. \\
& \left.\quad+\left\|\mathbf{u}_{1}(s)-\mathbf{u}_{2}(s)\right\|_{\mathcal{V}}+\left\|\theta_{1}(s)-\theta_{2}(s)\right\|_{L^{2}(\Omega)}+\left\|\varsigma_{1}(s)-\varsigma_{2}(s)\right\|_{L^{2}(\Omega)}\right) d s \\
& \quad+C\left(\left\|p\left(u_{1 \eta \nu}-g\right)-p\left(u_{2 \eta \nu}-g\right)\right\|_{L^{2}\left(\Gamma_{3}\right)}\right.
\end{aligned}
$$

From (23) we can rewrite

$$
\begin{aligned}
&\left\|\Lambda_{0}\left(\eta_{1}(t), \lambda_{1}(t), \mu_{1}(t)\right)-\Lambda_{0}\left(\eta_{2}(t), \lambda_{2}(t), \mu_{2}(t)\right)\right\|_{\mathcal{V}^{\prime}} \\
& \leq L_{\mathcal{E}}\left\|\mathbf{u}_{1}(t)-\mathbf{u}_{2}(t)\right\| \mathcal{V}+L_{\mathcal{G}} \int_{0}^{t}\left(\left\|\sigma_{1}(s)-\sigma_{2}(s)\right\|_{\mathcal{H}}\right. \\
&+L_{\mathcal{A}}\left\|\dot{\mathbf{u}}_{1}(s)-\dot{\mathbf{u}}_{2}(s)\right\|_{\mathcal{V}}+\left\|\mathbf{u}_{1}(s)-\mathbf{u}_{2}(s)\right\|_{\mathcal{V}} \\
&\left.+\left\|\theta_{1}(s)-\theta_{2}(s)\right\|_{L^{2}(\Omega)}+\left\|\varsigma_{1}(s)-\varsigma_{2}(s)\right\|_{L^{2}(\Omega)}\right) d s \\
&+C\left(\left\|\mathbf{u}_{1}(t)-\mathbf{u}_{2}(t)\right\|_{\mathcal{V}}\right) \quad \text { a.e. } t \in(0, T) .
\end{aligned}
$$

On the other hand, since $\mathbf{u}_{i}(t)=\mathbf{u}_{0}+\int_{0}^{t} \dot{\mathbf{u}}_{i}(s) d s$, we know that for a.e. $t \in$ $(0, T)$,

$$
\left\|\mathbf{u}_{1}(t)-\mathbf{u}_{2}(t)\right\| \mathcal{V} \leq \int_{0}^{t}\left\|\dot{\mathbf{u}}_{1}(s)-\dot{\mathbf{u}}_{2}(s)\right\| \mathcal{V} d s .
$$

Applying Young's and Holder's inequalities, (69) becomes, via (70)

$$
\begin{aligned}
& \left\|\Lambda_{0}\left(\eta_{1}(t), \lambda_{1}(t), \mu_{1}(t)\right)-\Lambda_{0}\left(\eta_{2}(t), \lambda_{2}(t), \mu_{2}(t)\right)\right\|_{\mathcal{V}^{\prime}}^{2} \\
& \leq C \int_{0}^{t}\left(\left\|\sigma_{1}(s)-\sigma_{2}(s)\right\|_{\mathcal{H}}^{2}+\left\|\dot{\mathbf{u}}_{1}(s)-\dot{\mathbf{u}}_{2}(s)\right\|_{\mathcal{V}}^{2}+\left\|\mathbf{u}_{1}(s)-\mathbf{u}_{2}(s)\right\|_{\mathcal{V}}^{2}\right. \\
& \left.+\left\|\theta_{1}(s)-\theta_{2}(s)\right\|_{V}^{2}+\left\|\varsigma_{1}(s)-\varsigma_{2}(s)\right\|_{V}^{2}\right) d s \quad \text { a.e.t } \in(0, T) .
\end{aligned}
$$

Furthermore, we find by taking the substitution $\eta=\eta_{1}, \eta=\eta_{2}$ in (47) and choosing $\mathbf{v}=\dot{\mathbf{u}}_{1}-\dot{\mathbf{u}}_{2}$ as test function

$$
\left\langle\rho\left(\ddot{\mathbf{u}}_{1}(t)-\ddot{\mathbf{u}}_{2}(t)\right)+A \dot{\mathbf{u}}_{1}(t)-A \dot{\mathbf{u}}_{2}(t), \dot{\mathbf{u}}_{1}(t)-\dot{\mathbf{u}}_{2}(t)\right\rangle \mathcal{V}^{\prime} \times \mathcal{V}
$$




$$
=\left\langle\eta_{2}(t)-\eta_{1}(t), \dot{\mathbf{u}}_{1}(t)-\dot{\mathbf{u}}_{2}(t)\right\rangle_{\mathcal{V}^{\prime} \times \mathcal{V}} \quad \text { a.e. } t \in(0, T) .
$$

By virtue of (18) and (24), this equation becomes

$$
\begin{aligned}
& \frac{\left(\rho^{*}\right)^{2}}{2} \frac{d}{d t}\left\|\dot{\mathbf{u}}_{1}(t)-\dot{\mathbf{u}}_{2}(t)\right\|_{H}^{2}+m_{\mathcal{A}}\left\|\dot{\mathbf{u}}_{1}(t)-\dot{\mathbf{u}}_{2}(t)\right\|_{\mathcal{V}}^{2} \\
& \leq\left\|\eta_{2}(t)-\eta_{1}(t)\right\| \mathcal{V}_{\mathcal{V}^{\prime}}\left\|\dot{\mathbf{u}}_{1}(t)-\dot{\mathbf{u}}_{2}(t)\right\| \mathcal{V} .
\end{aligned}
$$

Integrating this inequality over the interval time variable $(0, t)$, Young inequality leads to

$$
\begin{aligned}
& \left(\rho^{*}\right)^{2}\left\|\dot{\mathbf{u}}_{1}(t)-\dot{\mathbf{u}}_{2}(t)\right\|_{H}^{2}+m_{\mathcal{A}} \int_{0}^{t}\left\|\dot{\mathbf{u}}_{1}(s)-\dot{\mathbf{u}}_{2}(s)\right\|_{\mathcal{V}}^{2} d s \\
& \leq \frac{2}{m_{\mathcal{A}}} \int_{0}^{t}\left\|\eta_{1}(s)-\eta_{2}(s)\right\|_{\mathcal{V}^{\prime}}^{2} d s .
\end{aligned}
$$

Consequently,

$$
\int_{0}^{t}\left\|\dot{\mathbf{u}}_{1}(s)-\dot{\mathbf{u}}_{2}(s)\right\|_{\mathcal{V}}^{2} d s \leq C \int_{0}^{t}\left\|\eta_{1}(s)-\eta_{2}(s)\right\|_{\mathcal{V}^{\prime}}^{2} d s \quad \text { a.e. } t \in(0, T) .
$$

which also implies, using a variant of (70), that

$$
\left\|\mathbf{u}_{1}(s)-\mathbf{u}_{2}(s)\right\|_{\mathcal{V}}^{2} \leq C \int_{0}^{t}\left\|\eta_{1}(s)-\eta_{2}(s)\right\|_{\mathcal{V}^{\prime}}^{2} d s \quad \text { a.e.t } \in(0, T),
$$

Moreover, if we take the substitution $\lambda=\lambda_{1}, \lambda=\lambda_{2}$ in (56) and subtracting the two obtained equations, we deduce by choosing $\omega=\theta_{\lambda_{1}}-\theta_{\lambda_{2}}$ as test function

$$
\begin{aligned}
& \frac{\left(\rho^{*}\right)^{2}}{2}\left\|\theta_{1}(t)-\theta_{2}(t)\right\|_{L^{2}(\Omega)}^{2}+C_{1} \int_{0}^{t}\left\|\theta_{1}(s)-\theta_{2}(s)\right\|_{V}^{2} d s \\
& \leq \int_{0}^{t}\left\|\lambda_{1}(s)-\lambda_{2}(s)\right\|_{V^{\prime}}\left\|\theta_{1}(s)-\theta_{2}(s)\right\|_{V} d s \quad \text { a.e. } t \in(0, T) .
\end{aligned}
$$

Employing Hölder's and Young's inequalities, we deduce that

$$
\begin{array}{r}
\left\|\theta_{\lambda_{1}}(t)-\theta_{\lambda_{2}}(t)\right\|_{L^{2}(\Omega)}^{2}+\int_{0}^{t}\left\|\theta_{\lambda_{1}}(s)-\theta_{\lambda_{2}}(s)\right\|_{V^{2}}^{2} d s \\
\leq C \int_{0}^{t}\left\|\lambda_{1}(s)-\lambda_{2}(s)\right\|_{V^{\prime}}^{2} d s \quad \text { a.e. } t \in(0, T) .
\end{array}
$$

Substituting now $\left\{\mu=\mu_{1}, \xi=\tilde{\varsigma}_{\mu_{1}}\right\},\left\{\mu=\mu_{2}, \xi=\tilde{\varsigma}_{\mu_{2}}\right\}$ in (61) and subtracting the two inequalities, we obtain

$$
\left\|\tilde{\varsigma}_{1}(t)-\tilde{\varsigma}_{2}(t)\right\|_{L^{2}(\Omega)}^{2}+\int_{0}^{t}\left\|\tilde{\varsigma}_{1}(t)-\tilde{\varsigma}_{2}(t)\right\|_{V}^{2} d s
$$




$$
\leq C \int_{0}^{t}\left\|e^{-k_{1} t}\left(\mu_{1}(s)-\mu_{2}(s)\right)\right\|_{V^{\prime}}^{2} d s \text { a.e. } t \in(0, T),
$$

from which also follows that

$$
\begin{aligned}
& \left\|\varsigma_{1}(t)-\varsigma_{2}(t)\right\|_{L^{2}(\Omega)}^{2}+\int_{0}^{t}\left\|\varsigma_{1}(s)-\varsigma_{2}(s)\right\|_{V}^{2} d s \\
& \leq C \int_{0}^{t}\left\|\mu_{1}(s)-\mu_{2}(s)\right\|_{V^{\prime}}^{2} d s \text { a.e. } t \in(0, T),
\end{aligned}
$$

We can infer, using (64), (71), (72), (74) and (75), that

$$
\begin{aligned}
\int_{0}^{t} & \left\|\Lambda_{0}\left(\eta_{1}(s), \lambda_{1}(s), \mu_{1}(s)\right)-\Lambda_{0}\left(\eta_{2}(s), \lambda_{2}(s), \mu_{2}(s)\right)\right\|_{\mathcal{V}^{\prime}}^{2} d s \\
\leq & C \int_{0}^{t} \int_{0}^{s}\left(\left\|\dot{\mathbf{u}}_{1}(r)-\dot{\mathbf{u}}_{2}(r)\right\|_{\mathcal{V}}^{2}+\left\|\theta_{1}(r)-\theta_{2}(r)\right\|_{V}^{2}\right. \\
& \left.+\left\|\mathbf{u}_{1}(r)-\mathbf{u}_{2}(r)\right\|_{\mathcal{V}}^{2}+\left\|\varsigma_{1}(r)-\varsigma_{2}(r)\right\|_{V}^{2}\right) d r d s \quad \text { a.e. } t \in(0, T) \\
\leq & C \int_{0}^{T} \int_{0}^{T}\left(\left\|\dot{\mathbf{u}}_{1}(r)-\dot{\mathbf{u}}_{2}(r)\right\|_{\mathcal{V}}^{2}+\left\|\theta_{1}(r)-\theta_{2}(r)\right\|_{V}^{2}\right. \\
& \left.+\left\|\mathbf{u}_{1}(r)-\mathbf{u}_{2}(r)\right\|_{\mathcal{V}}^{2}+\left\|\varsigma_{1}(r)-\varsigma_{2}(r)\right\|_{V}^{2}\right) d r d s \\
\leq & C \int_{0}^{T}\left(\left\|\dot{\mathbf{u}}_{1}(s)-\dot{\mathbf{u}}_{2}(s)\right\|_{\mathcal{V}}^{2}\right. \\
& \left.+\left\|\mathbf{u}_{1}(s)-\mathbf{u}_{2}(s)\right\|_{\mathcal{V}}^{2}+\left\|\theta_{1}(s)-\theta_{2}(s)\right\|_{V}^{2}+\left\|\varsigma_{1}(s)-\varsigma_{2}(s)\right\|_{V}^{2}\right) d s \\
\leq & C \int_{0}^{T}\left(\left\|\eta_{1}(s)-\eta_{2}(s)\right\|_{\mathcal{V}^{\prime}}^{2}+\left\|\lambda_{1}(s)-\lambda_{2}(s)\right\|_{V^{\prime}}^{2}+\left\|\mu_{1}(s)-\mu_{2}(s)\right\|_{V^{\prime}}^{2}\right. \\
& \left.+\left\|\mathbf{u}_{1}(s)-\mathbf{u}_{2}(s)\right\|_{\mathcal{V}}^{2}\right) d s
\end{aligned}
$$

Thus, by (73), we find

$$
\begin{aligned}
& \int_{0}^{T}\left\|\Lambda_{0}\left(\eta_{1}(s), \lambda_{1}(s), \mu_{1}(s)\right)-\Lambda_{0}\left(\eta_{2}(s), \lambda_{2}(s), \mu_{2}(s)\right)\right\|_{\mathcal{V}^{\prime}}^{2} d s \\
& \leq C \int_{0}^{T}\left(\left\|\eta_{1}(s)-\eta_{2}(s)\right\|_{\mathcal{V}^{\prime}}^{2}+\left\|\lambda_{1}(s)-\lambda_{2}(s)\right\|_{V^{\prime}}^{2}+\left\|\mu_{1}(s)-\mu_{2}(s)\right\|_{V^{\prime}}^{2}\right) d s .
\end{aligned}
$$

Furthermore, hypothesis (21) implies

$$
\int_{0}^{t}\left\|\psi\left(\sigma_{1}(s), \varepsilon\left(\dot{\mathbf{u}}_{1}(s)\right), \theta_{1}(s), \varsigma_{1}(s)\right)-\psi\left(\sigma_{2}(s), \varepsilon\left(\dot{\mathbf{u}}_{2}(s)\right), \theta_{2}(s), \varsigma_{2}(s)\right)\right\|_{V^{\prime}}^{2} d s
$$




$$
\begin{aligned}
\leq & 3 L_{\psi}^{2} \int_{0}^{t}\left(\left\|\sigma_{1}(s)-\sigma_{2}(s)\right\|_{\mathcal{H}}^{2}+\left\|\dot{\mathbf{u}}_{1}(s)-\dot{\mathbf{u}}_{2}(s)\right\|_{\mathcal{V}}^{2}\right. \\
& \left.+\left\|\theta_{1}(s)-\theta_{2}(s)\right\|_{V}^{2}+\left\|\varsigma_{1}(t)-\varsigma_{2}(t)\right\|_{V}^{2}\right) d s \quad \text { a.e. } t \in(0, T)
\end{aligned}
$$

This permits us to deduce, via (64), (72), (74) and (75), that

$$
\begin{aligned}
& \int_{0}^{T}\left\|\psi\left(\sigma_{1}(s), \varepsilon\left(\dot{\mathbf{u}}_{1}(s)\right), \theta_{1}(s), \varsigma_{1}(s)\right)-\psi\left(\sigma_{2}(s), \varepsilon\left(\dot{\mathbf{u}}_{2}(s)\right), \theta_{2}(s), \varsigma_{2}(s)\right)\right\|_{V^{\prime}}^{2} d s \\
& \leq C \int_{0}^{T}\left(\left\|\eta_{1}(s)-\eta_{2}(s)\right\|_{\mathcal{V}^{\prime}}^{2}+\left\|\lambda_{1}(s)-\lambda_{2}(s)\right\|_{V^{\prime}}^{2}+\left\|\mu_{1}(s)-\mu_{2}(s)\right\|_{V^{\prime}}^{2}\right) d s
\end{aligned}
$$

Similarly, using (64), (73), (74) and (75), we obtain the following estimate for $\phi$,

$$
\begin{aligned}
& \int_{0}^{T}\left\|\phi\left(\sigma_{1}(s), \varepsilon\left(\mathbf{u}_{1}(s)\right), \theta_{1}(s), \varsigma_{1}(s)\right)-\phi\left(\sigma_{2}(s), \varepsilon\left(\mathbf{u}_{2}(s)\right), \theta_{2}(s), \varsigma_{2}(s)\right)\right\|_{V^{\prime}}^{2} d s \\
& \leq C \int_{0}^{T}\left(\left\|\eta_{1}(s)-\eta_{2}(s)\right\|_{\mathcal{V}^{\prime}}^{2}+\left\|\lambda_{1}(s)-\lambda_{2}(s)\right\|_{V^{\prime}}^{2}+\left\|\mu_{1}(s)-\mu_{2}(s)\right\|_{V^{\prime}}^{2}\right) d s .
\end{aligned}
$$

From (76), (77) and (78), we conclude that there exists a positive constant $C>0$ verifying

$$
\begin{aligned}
& \left\|\Lambda\left(\eta_{1}, \lambda_{1}, \mu_{1}\right)-\Lambda\left(\eta_{2}, \lambda_{2}, \mu_{2}\right)\right\|_{L^{2}\left(0, T ; \mathcal{V}^{\prime} \times V^{\prime} \times V^{\prime}\right)} \\
& \leq C\left\|\left(\eta_{1}-\eta_{2}, \lambda_{1}-\lambda_{2}, \mu_{1}-\mu_{2}\right)\right\|_{L^{2}\left(0, T ; \mathcal{V}^{\prime} \times V^{\prime} \times V^{\prime}\right)},
\end{aligned}
$$

and so, by reapplication of mapping $\boldsymbol{\Lambda}$, yields

$$
\begin{aligned}
& \left\|\Lambda^{2}\left(\eta_{1}, \lambda_{1}, \mu_{1}\right)-\Lambda^{2}\left(\eta_{2}, \lambda_{2}, \mu_{2}\right)\right\|_{L^{2}\left(0, T ; \mathcal{V}^{\prime} \times V^{\prime} \times V^{\prime}\right)} \\
& \leq \frac{C^{2}}{2 !}\left\|\left(\eta_{1}-\eta_{2}, \lambda_{1}-\lambda_{2}, \mu_{1}-\mu_{2}\right)\right\|_{L^{2}\left(0, T ; \mathcal{V}^{\prime} \times V^{\prime} \times V^{\prime}\right)} .
\end{aligned}
$$

We generalize this procedure by recurrence on $n$. Then we obtain the formula

$$
\begin{aligned}
& \left\|\Lambda^{n}\left(\eta_{1}, \lambda_{1}, \mu_{1}\right)-\Lambda^{n}\left(\eta_{2}, \lambda_{2}, \mu_{2}\right)\right\|_{L^{2}\left(0, T ; \mathcal{V}^{\prime} \times V^{\prime} \times V^{\prime}\right)} \\
& \leq \frac{C^{n}}{n !}\left\|\left(\eta_{1}-\eta_{2}, \lambda_{1}-\lambda_{2}, \mu_{1}-\mu_{2}\right)\right\|_{L^{2}\left(0, T ; \mathcal{V}^{\prime} \times V^{\prime} \times V^{\prime}\right)} .
\end{aligned}
$$

We know that the sequence $\left(C^{n} / n !\right)_{n}$ converges to 0 . So, for $n$ sufficiently large $\frac{C^{n}}{n !}<1$. It means that a large power $n$ of the operator $\Lambda$ is a contraction on $L^{2}\left(0, T ; \mathcal{V}^{\prime} \times V^{\prime} \times V^{\prime}\right)$. Hence, Banach fixed point theorem shows that $\Lambda$ admits a unique fixed point $\left(\eta^{*}, \lambda^{*}, \mu^{*}\right) \in L^{2}\left(0, T ; \mathcal{V}^{\prime} \times V^{\prime} \times V^{\prime}\right)$. 
We can now prove the existence of a solution to problem (PV). To this aim, it is sufficient to remark that for a.e. $t \in(0, T)$,

$$
\begin{gathered}
\left(\eta^{*}(t), \mathbf{v}\right)_{\mathcal{V}, \mathcal{V}}=\left(\mathcal{E}\left(\varepsilon\left(\mathbf{u}_{\eta^{*}}(t)\right)\right), \varepsilon(\mathbf{v})\right)_{\mathcal{H}} \\
+\left(\int_{0}^{t} \mathcal{G}\left(\sigma_{\eta^{*}, \lambda^{*}, \mu^{*}}(s)-\mathcal{A}\left(\varepsilon\left(\dot{\mathbf{u}}_{\eta^{*}}(s)\right)\right), \varepsilon\left(\mathbf{u}_{\eta^{*}}(s)\right), \theta_{\lambda^{*}}(s), \varsigma_{\mu^{*}}(s)\right) d s, \varepsilon(\mathbf{v})\right)_{\mathcal{H}} \\
+j(\mathbf{u}(t), \mathbf{v}) \forall \mathbf{v} \in \mathcal{V} \\
\lambda^{*}(t)=\psi\left(\varepsilon\left(\mathbf{u}_{\eta^{*}}(t)\right), \theta_{\lambda^{*}}(t), \varsigma_{\mu^{*}}(t)\right) \\
\mu^{*}(t)=\phi\left(\varepsilon\left(\mathbf{u}_{\eta^{*}}(t)\right), \theta_{\lambda^{*}}(t), \varsigma_{\mu^{*}}(t)\right)
\end{gathered}
$$

The uniqueness part of Theorem 1 is a consequence of the uniqueness of the fixed point of the operators defined by (67) and (68) and the unique solvability of the problems $\mathrm{PV}_{\eta}, \mathrm{PV}_{\lambda}, \mathrm{PV}_{\mu}$ and $\mathrm{PV}_{\eta, \lambda, \mu}$ which completes the proof of theorem 1.

Theorem 2 (Positivity of the temperature). Let the hypotheses of Theorem 1 hold and suppose in addition that

$$
\begin{gathered}
\psi(\sigma, \varepsilon(\mathbf{u}), \theta, \varsigma) \geq 0 \quad \text { a.e. in } \Omega \times(0, T), \\
q \geq 0 \quad \text { a.e. in } \Omega \times(0, T), \\
\theta_{0} \geq 0 \quad \text { a.e. in } \Omega \times(0, T) .
\end{gathered}
$$

Then, the solution $\{\mathbf{u}, \sigma, \theta, \varsigma\}$ to problem $(P V)$ satisfies the following property

$$
\theta(x, t) \geq 0 \quad \text { for a.e. }(x, t) \in \Omega \times(0, T) .
$$

Proof. We use a maximum principle argument [5]. Thus, we test the equation (35) by the function $-\theta^{-}$, where $\theta^{-}=\max \{0,-\theta\}$, and integrate over $(0, T)$. By using the hypothesis (81), (82) and (83), we have

$$
\begin{aligned}
& \frac{1}{2}\left(\rho^{*}\right)^{2}\left\|\theta^{-}\right\|_{L^{\infty}\left(0, T ; L^{2}(\Omega)\right)}^{2}+C_{1}\left\|\theta^{-}\right\|_{L^{2}(0, T ; V)}^{2} \\
& \leq-\int_{0}^{t} \int_{\Omega} \psi(\varepsilon(\mathbf{u}(x, s)), \theta(x, s), \varsigma(x, s)) \theta^{-}(x, s) d x d s \\
& \quad-\int_{0}^{t} \int_{\Omega} q(x, s) \theta^{-}(x, s) d x d s \leq 0 \quad \text { a.e. } t \in(0, T) .
\end{aligned}
$$

it ressults that $\left\|\theta^{-}\right\|_{L^{2}(0, T ; V) \cap L^{\infty}\left(0, T ; L^{2}(\Omega)\right)} \leq 0$. Then $\theta^{-}=0$ which prouves the positivity of the temperature. 


\section{References}

[1] A. Amassad, M Shillor and M. Sofonea, A quasistatic contact problem for an elastic perfectly plastic body with Tresca's friction, Nonlin. Anal, (35) (1999), 95-105.

[2] A. Amassad, C. Fabre and M. Sofonea; A Quasistatic Viscoplastic Contact Problem with Normal Compliance and Friction, IMA Journal of Applied Mathematics 69 (2004), 463-482.

[3] K. T. Andrews, A. Klarbring, M. Shillor and S. Wright; A Dynamic Thermoviscoelastic Contact Problem with Friction and Wear, Int. J. Eng. Sci, Vol 35, No 14, 1291-1309. (1997).

[4] Y. Ayyad and M. Sofonea; Analysis of Tow Dynamic Frictionless Contact Problems for Elastic-Visco-Plastic Materials, Electronic Journal of Differential Equations, Vol. 2007(2007), No. 55, pp. 1-17.

[5] C. Baiocchis and A. Capelo; Variational and Quasivariational Inequalities: Application to Free Boundary Problems, Wiley-Interscience, ChichesterNew York; 1984.

[6] E. Bonetti and G. Bonfanti; Existence and Uniqueness of the Solution to $3 D$ Thermoviscoelastic System, Electronic Journal of Differential Equations, Vol. 2003, No. 50, pp. 1-15.

[7] H. Brezis; Equations et Inéquations Non Linéaires dans les Espaces en Dualité, Annale de l'Institut Fourier, Tome 18, n¹, (1968), p. 115-175.

[8] N. Crestescu; Elastic/Viscoplastic Constitutive Equations for Rocks, Int : J. Roch, Mech. Min. Sci \& Geomech. Abster 24 (5), 271-282 (1987).

[9] O. Chau, J.R. Ferndez, M. Shillor, M. Sofonea; Variational and numerical analysis of a quasistatic viscoelastic contact problem with adhesion, J. Comput. Appl. Math, 159, pp. 431-465, 2003.

[10] O. Chau, M. Shillor, M. Sofonea; Dynamic frictionless contact with adhesion, Z. Angew. Math.Phys., 55, pp. 32-47, 2004.

[11] N. Cristescu and I. Suliciu; Viscoplasticity, Martinus Nijhoff Publishers, Editura Tehnica, Bucharest, (1982).

[12] G. Duvaut and J. L. Lions; Les Inéquations en Mécanique et en Physique, Dunod (1976). 
[13] J. R. Fernández-García, M. Sofonea and J. M. Viaňo; A Frictionless Contact Problem for Elastic-Viscoplastic Materials with Normal Compliance, Numerische Mathemätik 90 (2002), 689-719.

[14] M. Frémond; Adhérence des solides, J. Mécanique Théorique et Appliquée, 6, pp. 383-407, 1987.

[15] M. Frémond; Equilibre des structures qui adhèrent à leur support C. R. Acad. Sci. Paris, Sér. II, 295, pp. 913-916, 1982.

[16] M. Frémond and B. Nedjar; Damage, Gradient of Damage and Principle of Virtual Work, Int. J. Solids Structures, 33 (8), 1083-1103. (1996).

[17] M. Frémond, K. L. Kuttler and M. Shillor; Existence and Uniqueness of Solution for a One-Dimensional Damage Model, J. Math. Anal. Appl. 229, 271-294. (1999).

[18] P. Germain; Cours de Mécanique des Milieux Continus, Masson et Cie, Paris, (1973).

[19] W. Han and M. Sofonea; Quasistatic Contact Problems in Viscoelasticity and Viscoplasticity, Studies in Advanced Mathematics 30, American Mathematical Society, Providence, RI-International Press, Somerville, MA, (2002).

[20] I. R. Ionescu and M. Sofonea; Functional and Numerical Methods in Viscoplasticity, Oxford University Press, Oxford, 1994.

[21] S. Kobayashi and N. Robelo; A Coupled Analysis of Viscoplastic Deformation and Heat Transfer: I Theoretical Consideration, II Applications, Int, J. of Mech. Sci, 22, 699-705, 707-718, (1980).

[22] K. L. Kuttler; Dynamic Frictional Contact for Elastic Viscoplastic Material, Electronic Journal of Differential Equations, Vol. 2007(2007), No. 75, pp. 1-20.

[23] J. L. Lions; Quelques Méthodes de Résolution des Problèmes Aux Limites Non Linéaires, Dunod (1969).

[24] J. L. Lions and E. Magénes; Problèmes aux Limites Non Homogènes et Applications, Volume I, Dunod (1968). 
[25] J. Nečas and J. Kratochvil; On Existence of the Solution Boundary Value Problems for Elastic-Inelastic Solids, Comment. Math. Univ. Carolinea, 14, 755-760, (1973).

[26] F. Messelmi and B. Merouani; Quasi-Static Evolution of Damage in Thermo-Viscoplastic Materials, Analele Universitătii Oradea, Fasc. Mathematica, Tome XVII (2010), Issue No. 2, 133-148.

[27] A. B Merouani, F.Messelmi; Dynamic Evolution of Damage in ElasticThermo-Viscoplastic Materials. Electronic Journal of Differential Equations, Vol. 2010(2010), No. 129, pp. 1-15.

[28] F. Messelmi, B. Merouani and M. Meflah; Nonlinear Thermoelasticity Problem, Analele Universităţii Oradea, Fasc. Mathematica, Tome XV (2008), 207-217.

[29] F. Messelmi, B. Merouani and F. Bouzeghaya; Steady-State Thermal Herschel-Bulkley Flow with Tresca's Friction Law, Electronic Journal of Differential Equations, Vol. 2010(2010), No. 46, pp. 1-14.

[30] M. Raous, L. Cangémi,M. Cocu; A consistent model coupling adhesion, friction, and unilateral contact, Comput. Methods Appl. Mech. Eng., 177, pp. 383-399, 1999.

[31] M. Rochdi, M. Shillor and M. Sofonea; Analysis of Quasistatic Viscoelastic Problem with Friction and Damage, Adv. Math. Sci. App. 10, 173-189. (2002).

[32] M. Rochdi, M. Shillor and M. Sofonea; A Quasistatic Viscoelastic Contact Problem with Normal Compliance and Friction, Journal of Elasticity 51 (1998), 105-126.

[33] J. Rojek, J.J. Telega; Contact problems with friction, adhesion and wear in orthopaedic biomechanics. I: General developments, J. Theor. Appl. Mech., 39, pp. 655-677, 2001.

[34] L. Selmani and M. Selmani; Analysis of a Viscoelastic Contact Problem with Normal Damped and Damage, Bull. Belg. Math. Soc. Simon Stevin Volume 13, Number 2 (2006), 209-220.

[35] M. Selmani, L. Selmani; Analysis of a frictionless contact problem for elastic-viscoplastic material.Nonlinear Analysis: Modelling and Control, 2012, Vol. 17, No. 1, 99-117 
[36] M. Shillor, M. Sofonea and J. J. Telega; Models and Analysis of Quasistatic Contact, Lecture Notes in Physics 655, Springer, Berlin, 2004.

[37] M. Sofonea; Quasistatic Processes for Elastic-Viscoplastic Materials with Internal State Variables, Annales Scientifiques de l'Université ClermontFerrand 2, Tome 94, Série Mathématiques, n 25. p. 47-60, (1989).

[38] M. Sofonea; Functional Methods in Thermo-Elasto-Visco-Plasticity, Ph. D. Thesis, Univ of Buccharest, (1988) (in Romanian). 
